\title{
On Chaotic Dynamics in Rational Polygonal Billiards
}

\author{
Valery B. KOKSHENEV
}

Departamento de Fisica, Universidade Federal de Minas Gerais, Instituto de Ciencias Exatas, Caixa Postal 702, CEP 30123-970, Belo Horizonte, MG, Brazil

E-mail: valery@fisica.ufmg.br

Received June 23, 2005, in final form October 29, 2005; Published online November 13, 2005

Original article is available at http://www.emis.de/journals/SIGMA/2005/Paper014/

\begin{abstract}
We discuss the interplay between the piece-line regular and vertex-angle singular boundary effects, related to integrability and chaotic features in rational polygonal billiards. The approach to controversial issue of regular and irregular motion in polygons is taken within the alternative deterministic and stochastic frameworks. The analysis is developed in terms of the billiard-wall collision distribution and the particle survival probability, simulated in closed and weakly open polygons, respectively. In the multi-vertex polygons, the late-time wall-collision events result in the circular-like regular periodic trajectories (sliding orbits), which, in the open billiard case are likely transformed into the surviving collective excitations (vortices). Having no topological analogy with the regular orbits in the geometrically corresponding circular billiard, sliding orbits and vortices are well distinguished in the weakly open polygons via the universal and non-universal relaxation dynamics.
\end{abstract}

Key words: polygons; hyperbolic systems with singularities; stochastic system; chaotic dynamics; anomalous diffusion process

2000 Mathematics Subject Classification: 37D45; 37D50; 51E12; 60C05; $60 \mathrm{~J} 60$

\section{Introduction}

Classical polygonal billiards, formed by the piecewise-linear billiard boundary with the vertex angles that are rational multiplies of $\pi$, are rational polygons, known as non-chaotic systems (see [1] for review). They are therefore quite distinct from the Sinai billiard [2] (SB) and the Bunimovich billiard [3] (BB), in which classical chaotic motion regimes are due to, respectively, dispersive effects caused by the boundary, formed by the disk and the square, and the interplay between boundary segments, formed by the semi-circles and the square. Moreover, rational polygons of $m$ equal sides and $m$ equal vertices (hereafter, $m$-gons [1]) exhibit chaotic-like changes in the associated quantum-level spectra [4], but the fluctuations found [5] are very close to those known in the universal Gaussian statistics. These controversial evidences for the chaotic and regular behavior of polygons are a challenge to dynamic theory of billiards. In the present paper we develop a physical insight into the problem, based on analysis of the simulation data on the billiard collision statistics. Within a more general context, the delicate problem of the interplay between regular and irregular segments, which constitute the total billiard boundary, is ultimately related to apparent controversy between the causality and randomness. Our approach to the problem is developed through the alternative deterministic and stochastic frameworks, which elucidate the duality of chaotic and non-chaotic features in the intrinsic dynamics of the rational polygons.

The singular effects, provoked by the vertex-angle splitting of orbits, is one of the major problem in dynamics of non-dispersed billiards [1]. Being countable, they cannot be described within the standard Markov partition scheme. Similarly to the recent study on the arc-touching effects in dispersing SB [6], we give analysis of vertex-splitting effects on the basis of the genera- 
lized diffusion equation. The issue of our investigation is the late-time dynamics memory effects induced by sides and vertices in both closed and the open rational polygons.

\section{Collision statistics}

\subsection{Collision distribution function}

In billiards formed by classical particles of unit mass, moving with unit velocities, the billiard collision distribution function $D(n, t)$ is determined as the probability for a given particle to undergo $n$ random collisions with the boundary in a time interval $t$ (for the rigorous definition of $D(n, t)$ and for the way of its numerical observation, see [7]). With accounting for the property of ergodicity, this provides the mean collision number

$$
n_{\mathrm{c}}(t) \equiv\langle n\rangle_{\mathrm{c}}=\int_{0}^{\infty} n D(n, t) d n=\int n(\boldsymbol{x}, t) d \mu(\boldsymbol{x})=\frac{t}{\tau_{\mathrm{c}}}, \quad t \gg \tau_{\mathrm{c}} .
$$

Here $n(\boldsymbol{x}, t)$ is the number of billiard collisions during time $t$, for a particle of the position set $\boldsymbol{x}=(x, y, \theta)$, which includes its location $(x, y)$ and the velocity launching angle $\theta=[0,2 \pi]$ counted of the $x$-axis of the billiard table in the space $\Omega$. The continues-time evolution of the particle orbits preserves the Liouville measure [8]

$$
d \mu(\boldsymbol{x})=\frac{1}{2 \pi A} d x d y d \theta .
$$

and establishes the mean collision time $[7,8,9]$

$$
\tau_{\mathrm{c}}=\frac{\pi A}{P},
$$

controlled by the accessible area $A$ and the perimeter $P$ of a billiard table. Such a description of the continuos dynamic system can be derived from the late time behavior of the associated collision subsystem $[7,8,10]$.

The case of $m$-gon is specified by circumscribing of the rational polygon below a circle of radius $r$. For a given $m$, the billiard area $A_{m}=\left(m r^{2} / 2\right) \sin (2 \pi / m)$ and the perimeter $P_{m}=$ $2 m r \sin (\pi / m)$ provide the mean collision time

$$
\tau_{\mathrm{cm}}=\frac{\pi r}{2} \cos \left(\frac{\pi}{m}\right) .
$$

In the 'infinite-vertex' $m$-gon limit, one naturally arrives at the circle-billiard (CB) of radius $r$, with the mean collision time

$$
\tau_{\mathrm{c} \infty}=\tau_{\mathrm{c}}^{(\mathrm{CB})}=\frac{\pi r}{2} .
$$

A general question arises how geometrical changes of the billiard boundary are followed by corresponding classical dynamics. This question is related to the problem of the existence of the correspondence principle in polygonal billiards [11]. In view of equation (5), it sounds as whether the $\infty$-gon is a dynamic analog of the integrable CB.

\subsection{Dynamic diffusion regimes}

We have communicated [12] that the chaotic disk-dispersing SBs and non-chaotic vertex-splitting polygonal billiards display a similar behavior attributed to the enhanced diffusion dynamics. In polygons, this late-time dynamics is associated with the singular arc-splitting effects, produced by singularities of orbits provoked by the vertices [1]. The stochastic approach proposed for 


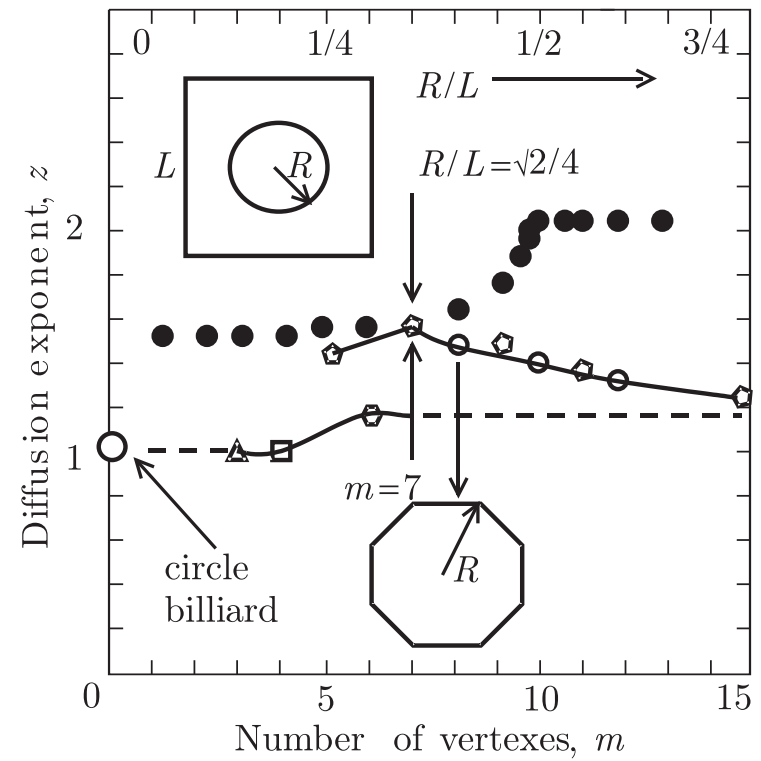

Figure 1. Observation of the diffusion dynamics in the Sinai (above) and polygonal (below) billiards. Points: closed circles - numerical data for the SBs of side $L=1$ for distinct dispersing disks of radii $R$. The open triangle, square, pentagon, etc. are the data for the regular polygons with $m=3,4,5$, etc. equal sides. The lines are a guide to the eye. The experimental error does not exceed the size of symbols.

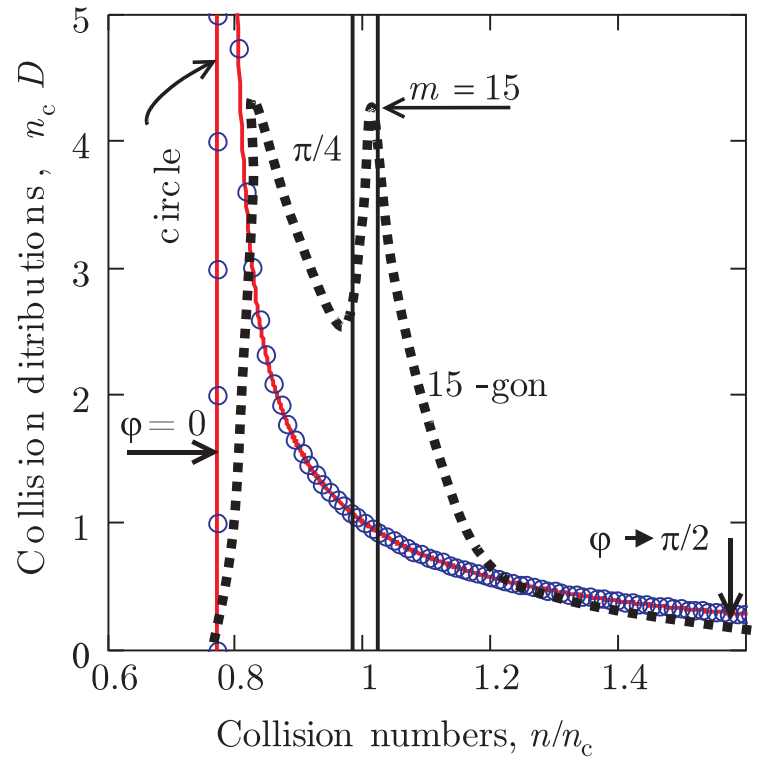

Figure 2. Observation of the collision distribution function. The solid lines are theoretical predictions for the CB (8) and the 15-gonal billiard, estimated in the deterministic-motion approximation. The open circles and the point-dotted line schematically represent numerical simulation of the late-time distributions. For the original data see Fig. 1 in [12].

analysis of these effects is based on exploration of the generalized diffusion equation, which manifests the dynamic relaxation of non-Markovian time evolution [13]. This fundamental model-independent equation, which generalizes the Brownian diffusion, is given for the variance of particle displacements $\Delta^{2} r(t)$ realized during time $t$. In application to $m$-gons, this equation was introduced through the variance of the number of random collisions [12], namely

$$
\Delta^{2} n_{\mathrm{cm}}(t) \equiv\left\langle\left(n-n_{\mathrm{cm}}\right)^{2}\right\rangle_{\mathrm{c}} \backsim\left(\frac{t}{\tau_{\mathrm{cm}}}\right)^{2 / z_{m}}, \quad t \gg \tau_{\mathrm{c}},
$$

represented with the help of equations (1) and (4). Here $z_{m}$ stands for the boundary-dependent diffusion exponent (which for the Brownian diffusion is equal to 2). In the case of chaotic SB, of side $L$ and of disk of radius $R$, the random walk treatment of the problem provided the equation [6]

$$
\Delta^{2} n_{\mathrm{c}}^{(\mathrm{SB})}(t) \equiv\left\langle\left(n-n_{\mathrm{c}}^{(\mathrm{SB})}\right)^{2}\right\rangle_{\mathrm{c}} \backsim\left[\frac{t}{\tau_{\mathrm{c}}(R)}\right]^{2 / z(R)}, \quad t \gg \tau_{\mathrm{c}}(R),
$$

corresponding to equation (6), taken with the mean collision time $\tau_{\mathrm{c}}(R)$, defined in equation (3), and the diffusion exponent $z(R)$. Equations (6) and (7) form a basis for the observation of distinct stationary regimes, driven by the billiard geometry. The uniform initial conditions were simulated by the standard random number generator RAN1 ${ }^{1}$ and the results of the analysis of temporal behavior of the directly observed variances for the billiard-wall number collisions are presented in Fig. 1.

\footnotetext{
${ }^{1}$ For details of the numerical experiments, see [10].
} 
In $\mathrm{SB}$, the universal ( $R$-independent) late-time diffusive regime is due to the stabilization of particle-displacement statistics with $z^{(\exp )}(R)=1.50 \pm 0.05$. This was rationalized [6] through the universal behavior of the particle propagator function [13] and the Lévy flights, which occur between the two distant scatterers, belonging to infinite Bleher's corridors of the dynamically equivalent Lorentz Gas (LG) [14]. This almost-free motion along the principal diagonal and non-diagonal corridors, induced by arc-touching dispersive events, terminates at $R \geq L \sqrt{2} / 4$, when the diagonal corridor becomes closed.

In $m$-gons, the non-universal dynamic regimes are well distinguished of those, observed in the chaotic SB and the non-chaotic $\mathrm{CB}$, having the unique ballistic regime defined by $z^{(\mathrm{SB})}(0)=$ $z^{(\mathrm{CB})}=1$. In the unilateral-triangle and square polygonal billiards, the vertex-splitting effects are fairly weak, because the regular orbits are due to the particles mainly move along the infinite trajectories, well known in the case $m=3$ [15]. As seen in Fig. 1, these particles expose their ballistic dynamics via $z_{3}^{(\exp )} \approx z_{4}^{(\exp )} \approx 1$, characteristic of the integrable CB. In contrast, the enhanced diffusion in pentagon and heptagon, developed by the vertex splitting of regular orbits, achieves its maximal level, indicated by the highest exponents $z_{5}^{(\exp )} \approx z_{7}^{(\exp )}=3 / 2$. In what follows, the observed distinct late-time dynamics is examined through the qualitative description of long-living orbits.

\subsection{Orbit topology}

It is disturbing that the question of whether every polygon has a periodic orbit remains open [1]. Meanwhile, any orbit, which is perpendicular to a side returns to this side parallel to itself and therefore it is periodic $[16,17]$. The existence of the perpendicular periodic trajectories in rational polygons was proved independently in [18] and [19]. The stability of the corresponding periodic orbits in arbitrary polygon was also shown [17].

In the integrable $\mathrm{CB}$ and in 'almost integrable' $m$-gons, limited by, say, $m=3,4$ and 6 , the launching angle $\theta(2)$ is almost good integral of motion for the particles randomly injected on the billiard ${ }^{1}$. Consequently, the orbit-set classification of the late-time living orbits in such non-chaotic billiards can be introduced through the collision angle $\varphi=[0, \pi / 2]$, estimated from the normal to the boundary wall $[10,20]$. This motion integral provides the basis for our deterministic approach to billiard dynamics proposed in [12] and then developed for the square billiard [6] and polygons [21].

In $\mathrm{CB}$, all regular orbits are ranged between the diameter-beating set and the 'whisperinggallery' sets established, respectively, by the collision angles $\varphi=0$ and $\varphi \approx \pi / 2$ (shown in Fig. 1). In triangle billiards, the existence of the 'perpendicular orbit' with $\varphi=0$ was suggested in [16], and then proved for any $m$-gon $[18,19]$. In the case of square billiard, these orbits correspond to the well-known 'bouncing-ball' orbits [10, 22, 23], given by $\varphi \approx 0$ - angle set and the $\varphi \approx \pi / 2$-set, moving in the horizontal and vertical directions, associated with the horizontal and vertical free-motion corridors. In the even- $m$-gons, the marginal vertex-to-vertex particle trajectories [1] formally correspond to the stable 'diameter-beating' orbits in CB. In contrast, the stable sliding orbits, observed for $m>4$, are treated as an analog of the whispering-gallery orbits in CB responsible for the late-time superdiffusive dynamics, revealed in Fig. 1 in the chaotic-like pentagon $\left(z_{5}^{(\exp )}=1.42\right)$ and in the ordered-like hexagon $\left(z_{6}^{(\exp )}=1.18\right)$. The chaotic effects are maximal in heptagon $\left(z_{7}^{(\exp )}=1.48\right)$, but with further increase the number of vertices, when $m \geq 8$, they attenuate gradually. The analysis shown in Fig. 1 suggests that in the 'infinite-vertex' limit, the superdiffusive motion is not transformed into the ballistic regime, i.e., $z_{\infty}>1$. Although the geometrical correspondence exists, as it is exposed in equation (5), the sliding orbits are topologically distinct from the whispering-gallery orbits in the $\mathrm{CB}$, and therefore the late-time dynamic correspondence between the $\infty$-gon and the CB does not exist. This statement is additionally tested in the next section. 


\subsection{Observation of orbits}

In $\mathrm{CB}$ of radius $r$, the collision distribution function

$$
D^{(\mathrm{CB})}(n, t)=\frac{\pi^{2} n_{\mathrm{c}}^{3}}{16 n^{4}}\left(1-\frac{\pi^{2} n_{\mathrm{c}}^{2}}{16 n^{2}}\right)^{-1 / 2}, \quad n_{\mathrm{c}}^{(\mathrm{CB})}=\frac{2 t}{\pi r}
$$

defined in equation (1), is found through the Liouville measure. Similarly, if one ignores the vertex-splitting (irregular) effects a given $m$-gon, the particle-collision function, corresponding to the regular orbits, can be obtained through the model (equivalent-side) approximation [21], namely

$$
\begin{aligned}
& D_{m}^{(\mathrm{reg})}(n, t)=\frac{\sin \varphi_{\mathrm{cm}}}{n_{\mathrm{cm}} \varphi_{\mathrm{cm}}^{2}}\left[1-\left(\frac{n}{n_{\mathrm{cm}}} \frac{\sin \varphi_{\mathrm{cm}}}{\varphi_{\mathrm{cm}}}\right)^{2}\right]^{-1 / 2}, \quad n_{\mathrm{cm}}=\frac{t}{\tau_{\mathrm{cm}}}, \\
& \text { for } \varphi_{\mathrm{cm}} \cot \varphi_{\mathrm{cm}} \leq n / n_{\mathrm{cm}} \leq \varphi_{\mathrm{cm}} / \sin \varphi_{\mathrm{cm}}, \quad \text { otherwise } \quad D_{m}^{(\mathrm{reg})}=0,
\end{aligned}
$$

where $\varphi_{\mathrm{cm}}=\pi / 2 m$, for the odd $m$-gon, and $\varphi_{\mathrm{cm}}=\pi / m$, for the even $m$-gon. A comparative analysis between the distributions observed in 15-gon and CB is displayed schematically in Fig. 2.

In Fig. 2, the diameter-beating orbit $(\varphi=0)$ and the whispering-gallery orbit $(\varphi \lesssim \pi / 2)$ are well observable in CB. In the case of 15-gon, the vertex-singular effects, manifested by deviations from the regular-orbit behavior, seem tend to be adapted through the bouncing-ball-like $(\varphi \approx 0)$ and the sliding orbits $(\varphi \approx \pi / 2)$. We see that both kinds of orbits with $\varphi \approx \pi / 2$ (shown by open circles and solid squares) are well distinguished, because, the sliding orbits are topologically distinct from their prototype in CB. This finding is supported by the distinct diffusion exponents, $z_{15}^{(\exp )}=1.2$ and $z^{(\mathrm{CB})}=1$, analyzed in Fig. 1 .

In a given $m$-gon, the characteristic collision time

$$
t_{\mathrm{cm}}^{(\mathrm{reg})}(\varphi)=\tau_{\mathrm{c} \infty} \frac{\sin \left(\varphi_{m}\right) \cos (\pi / m)}{\varphi_{m} \cos \left(\varphi-\psi_{m}\right)},
$$

was also found $[12,21]$, in the regular-orbit approximation. Here $\psi_{m}=\varphi_{\mathrm{cm}}$ and $\psi_{m}=0$, for, respectively, the even m-gon and odd m-gon cases, discussed in equation (9). One can see that $t_{\mathrm{cm}}(\varphi) \gg \tau_{\mathrm{cm}}$ for the set $\varphi \approx \pi / 2$. The longest-living regular (sliding) orbits are therefore expected to be observed in the weakly open $m$-gons.

\section{Surviving dynamics}

In the case of weakly open $m$-gons, one deals with $N(0)=10^{6}$ uniformly distributed point particles, which are allowed to escape through a small opening in the billiard boundary ${ }^{1}$. A temporal behavior of $N(t)$ non-escaped orbits (particles) is commonly scaled by the mean escape time $[7,10,22,24]$

$$
\tau_{\mathrm{e}}=\tau_{\mathrm{c}} \frac{P}{\Delta} \quad \text { with } \quad \Delta \ll P,
$$

where the mean collision time $\tau_{\mathrm{c}}$ is given in equation (3). The late-time asymptote of the billiard survival probability

$$
S(t)=\frac{N(t)}{N(0)} \propto\left(\frac{\tau_{\mathrm{e}}}{t}\right)^{\delta}, \quad \text { for } \quad t \gg \tau_{e},
$$

describing the algebraic-behavior through the decay dynamic exponent $\delta$. In non-chaotic square billiard, the algebraic decay with the exponents $\delta \lesssim 1$ was first reported in [22]. A careful study 


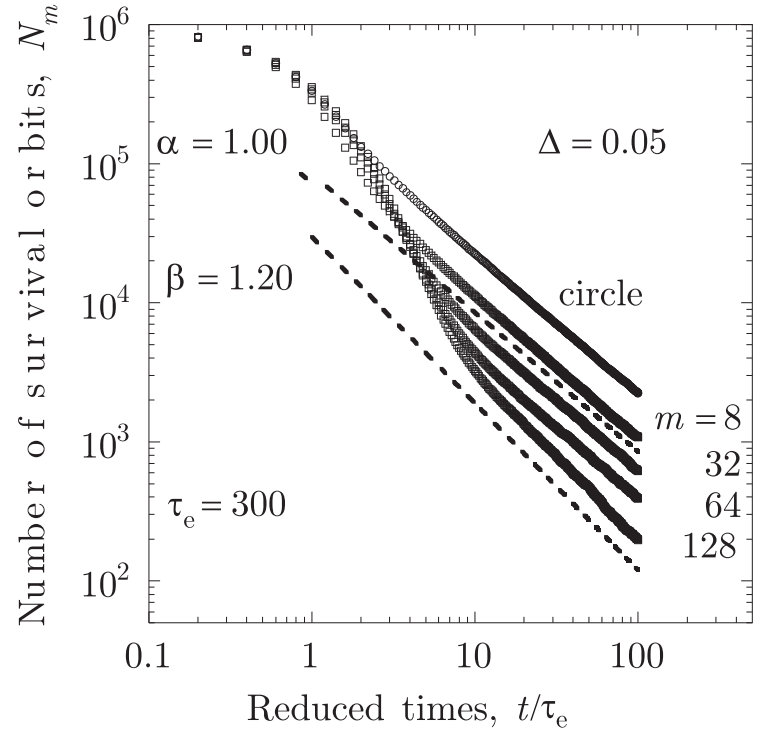

Figure 3. Analysis of the temporal evolution of the survived orbits in rational polygons with small opening width $\Delta=0.05 R$. The observation times are reduced through the escape characteristic time $\tau_{\mathrm{e}}=300$, chosen common for all cases. Points: numerical data for the simulated $m$-gons (squares) and the corresponding CB (circles).

of decay dynamics in the integrable CB and in the 'almost integrable' 4-gon established [10] two distinct channels of slow relaxation. The first channel is due to the regular-orbit motion with the decay exponent $\delta=1$, provided by the whispering-gallery orbits, and the secondary channel originates from the bouncing-ball orbits, exposed [10] by $\delta<1$.

In chaotic closed and weakly open classical systems (including Hamiltonian systems), exemplified by $\mathrm{BB}[23,24,25]$, infinite-horizon SB [20, 26], and by corresponding low-density LG $[14,27]$, the overall algebraic decay was found numerically through the geometry-dependent exponents $\delta \geq 1$. The channel of algebraic- type relaxation with $\delta=1$, common for both chaotic $[20,24,26]$ and non-chaotic $[10,22]$ billiards, seems to be generic for all incompletely hyperbolic systems with smooth convex boundaries. Its independence with respect to the billiard spatial dimension [26], its insensitivity to the details of boundary shape [10], including the location of a small opening [24], and to the initial conditions [10] suggests that the late-time $\alpha$-relaxation in classical systems is the universal primary relaxation. Unlike the primary relaxation, the secondary relaxation in chaotic billiards with $\delta>1$, is shown to be rather sensitive to the billiard geometry [10], as well as to the billiard table dimension $d$ [26], and to the initial conditions [10]. Example is the dynamic-exponent constraint $1<\beta \leq d$ estimated [26] and observed in both chaotic BB [24] and SB [26, 28]. As a result, the dynamic-regime classification can be proposed for the open classical billiards in terms of the decay dynamic exponent (12), namely

primary channel: $\quad \delta=\alpha=1, \quad$ universal regime;

secondary channel: $\delta=\beta \begin{cases}<1, & \text { non-chaotic regime, } \\ >1, & \text { chaotic regime. }\end{cases}$

In Fig. 3 we study the decay dynamics in $m$-gons with $m \geq 8$ through the number of survived particles $(12) N_{m}(t)=S_{m}(t) N_{m}(0)$. For the particular case of small opening $\Delta=0.05 R$, the universal relaxation (13) remains stable until $m=64$, but when $m \geq m_{\alpha}^{(\exp )}=128$ the primary-relaxation observation window becomes closed. The sliding regular-orbit dynamics likely transforms into a singular-orbit chaotic-like motion, when intensively affected by vertices. This is indicated by the dynamic decay exponent $\beta_{m}^{(\exp )}=1.2$ and equation (13). Also, the experimental observations was elaborated for the large opening $\Delta=0.20 R$, when $\beta_{m}^{(\exp )}=1.1$ and $m_{\alpha}^{(\exp )}=32$ were derived in this case (see Fig. 3 in [21]). We examine that the upper limit for the $\alpha$-channel-observation window shows its sensitivity to the opening width. This suggests that the primary relaxation dominates in $m$-gons with $3 \leq m<m_{\alpha}^{(\exp )}$, where the regular-orbit 
decay motion is established by the universal decay exponent $\delta_{m}=\alpha=1$. The latter is due to the 'perpendicular' and sliding orbits, which exhibit, respectively, the non-chaotic and chaotic behavior, which are not distinguished within this channel. In order to prove that the observed chaotic-like regime in the open system is really caused by the long-living sliding orbits, we deduce the observation conditions for such a decay dynamics.

Similarly to the boundary case $R=L \sqrt{2} / 4$, corresponding to the universal-to-non-universal crossover in relaxation dynamics of the chaotic SB (shown in Fig. 1 ), the $\alpha$-to- $\beta$ crossover occurs in $m$-gons with $m=m_{\alpha}$ and is associated with the transformation of the particle motion, induced by regular segments of boundary, into that provoked by its singular parts. Just as the periodic motion in the crystalline lattice is observed through the collective excitations (phonons), so the exploration by particles of translational periodicity of the billiard table with large $m$ can provide the collective-particle motion of characteristic time $t_{\mathrm{cm}}^{(\mathrm{coll})}(\varphi)=\tau_{\mathrm{c} \infty} \cos ^{-1}(\varphi)$, permitted for any regular orbit, preserving the collision angle $\varphi$. This qualitative estimate, following from equation (10) taken at $m \gg 1$, indicates that the idealized sliding orbits, observed now at $\varphi \approx \pi / 2$ and $m \gg 1$, leave the piecewise-linear part of the billiard boundary. The real sliding-orbit excitations of finite relaxation times $\tau_{\mathrm{cm}}^{(\mathrm{slide})}$, are thought as marginal regular orbits established by the maximal collision angles $\varphi_{m}^{\text {(slide) }}=\varphi_{m}(10)$ with $\tau_{\mathrm{cm}}^{\text {(slide) }}=t_{\mathrm{cm}}^{\text {(coll })}\left(\varphi_{m}\right)$. In contrast to the ideal sliding orbits, we also determine the ideal vortex orbits as sliding along the piecewise-linear part of the boundary without reflection. Such irregular 'orbits' are formally distinguished by the collision angles $\varphi_{m}^{\text {(vort) }}=\Phi_{m} / 2$, where $\Phi_{m}$ are rational vertex angles. In view of the $m$-fold rotational symmetry of the billiard table, the existence of the real singularlike vortex excitations (or vortices) is expected from the preservation of the angular momentum for a certain set of vertex-angle correlated orbits. We therefore assume that the real longliving sliding (regular) orbits are precursors of the vortex-like (singular) orbits, attributed to the relaxation times $\tau_{\mathrm{cm}}^{\text {(vort) }}=t_{\mathrm{cm}}^{(\text {coll })}\left(\Phi_{m} / 2\right)$. In this way, the $\alpha$-to- $\beta$-relaxation crossover is attributed to the observed regular-to-singular sliding-orbit transformation, that occurs starting from the large numbers $m_{\alpha}^{(\exp )}$. The observation condition for the crossover between the two distinct real dynamic regimes can be approximated by the condition of disappearance of the ideal sliding orbits above $m_{\alpha}$ and that of the ideal vortex orbits below $m_{\alpha}$.

A small opening of width $\Delta\left(\ll P_{m}\right)$ can be arranged at any point of boundary [24]. The favorable survival situation in the $\alpha$-relaxation regime, induced by the regular part of the boundary of length $L_{m}=P_{m} / m$, must exclude the vertex-angle effects under the constraint $m<m_{\alpha}$. The geometrical condition, at which the ideal vortices effectively escape from the billiard table, corresponds to the opening of width $\Delta \ll L_{m}$, located at one of the angle vertices. Conversely, in the late-time $\beta$-relaxation regime, the regular orbits do not survive at all, if any side is absorbed by the opening, i.e. when $\Delta \gg L_{m}$, with $m>m_{\alpha}$. Hence, the $\alpha$-to- $\beta$ crossover in relaxation is qualitatively ensured by the condition $\Delta=L_{m}$ at $m=m_{\alpha}$. Taking into consideration that in polygons with large number of sides $P_{m} \approx P_{\infty}=2 \pi r$, one obtains the desirable criterion of the $\alpha$-to- $\beta$ crossover (13), namely

$$
m_{\alpha}=\frac{2 \pi r}{\Delta}
$$

The experimental data $m_{\alpha}^{(\exp )}=128$ and 32 , discussed above, are in agreement with the estimates $m_{\alpha}=126$ and 31, following from equation (14). This finding justifies our surmise that the observed long-living vortices in $\beta$-relaxation are due to the sliding orbits modified by intensive vertex splitting. Finally, as follows from equation (14) in the limit $\Delta \rightarrow 0$ and as experimentally supported in Fig. 1, the observation window for vortices disappears in closed polygons. 


\section{Conclusion}

We have rationalized the late-time relaxation dynamics, observed in rational polygons, in terms of the so-called sliding orbits, associated with the periodic trajectories. The dynamic properties of the corresponding orbits, determined by the collision-angle set close to $\pi / 2$, are shown to be due to the interplay between the piece-line (regular) part of the billiard boundary and its vertexangle (singular) part. The sliding orbits are beyond the simplified polygonal-orbit classification by Gutkin [1], where the regular orbits are presented by the 'infinite-past-to-infinite-future' trajectories, and the 'mild discontinuity', caused by vertex-splitting effects, is due to the 'infinitepast-to-vertex', 'vertex-to-infinite-future', and 'vertex-to-vertex' marginal trajectories. Also, they are in a certain way opposed to the perpendicular orbits, rigorously studied in $[17,18,19]$. The experimental prove for the existence of the long-living periodic sliding trajectories challenges to further mathematical research in polygons.

The sliding orbits are well pronounced in simulated dynamics in both closed and open rational polygons. When the number of vertices is small $(m<5)$, these orbits are ineffective in the dynamics driving by the long-living bouncing-ball-like orbits. With increasing of the number of sides (and vertices), the sliding orbits become stable and therefore regular, but simultaneously expose chaotic-like features, in a way similar to that in the chaotic SB. These features have been revealed here through the diffusion exponent and the collision distribution function. The chaotic-like sliding orbits, nevertheless, play the role of regular whispering-gallery orbits, known in the integrable CB.

In the open $m$-gons, the chaotic properties of the long-living sliding orbits remain masked and hidden, when observed via the primary relaxation within the domain $8 \leq m<m_{\alpha}(\Delta)$. This situation changes above the $\alpha$-to- $\beta$ crossover, where new collective excitations, denominated by vortices, become to be pronounced in the secondary billiard relaxation. These chaotic excitations are associated with the collective vertex-splitting effects, established in multi-vertex $m$-gons, which number of vertices exceeds $m_{\alpha}=2 \pi r / \Delta$.

Regardless the proposed rationalization of the regular-like sliding orbits and the irregular-like vortices, we have experimentally revealed that the boundary-collision events, observed in the collective dynamics in rational polygons are dual of regular and singular of isolated particle collisions with the corresponding parts of the billiard boundary. Being related to the zeromeasure singularities in phase space, these effects violate the integrability of polygons [1], as well as the classical-to-quantum correspondence principle [12]. The latter finding is due to the memory of vertex-splitting effects, which do not disappear in the billiard dynamics when $m \rightarrow \infty$, and thereby forbid the interchange of temporal $(t \rightarrow \infty)$ and the spatial $(m \rightarrow \infty)$ limits.

\section{Acknowledgments}

The financial support of the Brazilian agency CNPq is acknowledged.

[1] Gutkin E., Billiards in polygons, J. Stat. Phys., 1996, V.83, 7-26.

[2] Sinai Y.G., Dynamical systems with elastic reflections. Ergodic properties of dispersing billiards, Russ. Math. Surv., 1970, V.25, 137-189.

[3] Bunimovich L.A., On ergodic properties of nowhere dispersing billiards, Commun. Math. Phys., 1979, V.65, 295-312.

[4] Cheon T., Cohen T.D., Quantum level statistics of pseudointegrable billiards, Phys. Rev. Lett., 1989, V.62, 2769-2772.

[5] Shimizu Y., Shudo A., Polygonal billiards: correspondence between classical trajectories and quantum eingenstates, Chaos, Solitons \& Fractals, 1995, V.5, 1337-1361.

[6] Kokshenev V.B., Vicentini E., Physical insight into superdiffusive dynamics of Sinai billiard through collision statistics, Phys. A, in press. 
[7] Garrido P.L., Gallavotti G., Billiards correlation functions, J. Stat. Phys., 1994, V.76, 549-585.

[8] Chernov N., Entropy, Lyapunov exponents, and mean free path for billiards, J. Stat. Phys., 1997, V.88, $1-29$.

[9] Garrido P.L., Kolmogorov-Sinai entropy, Lyapunov exponents, and mean free time in billiard systems, J. Stat. Phys., 1997, V.88, 807-825.

[10] Vicentini E., Kokshenev V.B., On survival dynamics of classical systems. Non-chaotic open billiards, Phys. A, 2001, V.295, 391-408.

[11] Mantica G., Quantum algorithmic integrability: the metaphor of classical polygonal billiards, Phys. Rev. E, 2001, V.61, 6434-6443.

[12] Kokshenev V.B., Vicentini E., From ballistic to Brownian motion through enhanced diffusion in vertexsplitting polygonal and disk-dispersing Sinai billiards, Phys. Rev. E, 2002, V.65, 015201-015204(R).

[13] Metzler R., Klafter J., The random walk's guide to anomalous diffusion: A fractional dynamics approach, Phys. Rep., 2000, V.339, 1-77.

[14] Bleher P.M., Statistical properties of two-dimensional periodic Lorentz gas with infinite horizon, J. Stat. Phys., 1992, V.66, 315-373.

[15] Veech W.A., Teichmuller curves in moduli space, Eisenstein series and an application to triangular billiards, Invent. Math., 1989, V.97, 553-583.

[16] Ruijgrok Th., Periodic orbits in triangular billiards, Acta Phys. Pol., 1991, V.22, 955-981.

[17] Troubetzkoy S., Recurrence and periodic billiard orbits in polygons, Reg. Chaotic Dyn., 2004, V.9, 1-12.

[18] Boshernitzan M., Billiards and rational periodic directions in polygons, Amer. Math. Monthly, 1992, 522529.

[19] Galperin G., Stepin A., Vorobets Ya., Periodic billiard trajectories in polygons: generating mechanisms, Russ. Math. Surv., 1992, V.47, 5-80.

[20] Kokshenev V.B., Nemes M.C., Escape probability for classically chaotic systems, Phys. A, 2000, V.275, 70-77.

[21] Kokshenev V.B., Vicentini E., Slow relaxation in weakly open rational billiards, Phys. Rev. E, 2003, V.68, 026221-026228

[22] Bauer W., Bertsch G.F., Decay of ordered and chaotic systems, Phys. Rev. Lett., 1990, V.65, 2213-2216.

[23] Pikovsky A.S., Escape exponent for transient chaos scattering in non-hyperbolic Hamiltonian systems, J. Phys. A: Math. Gen., 1992, V.25, L477-L481.

[24] Alt H., Gräf H.-D., Harney H.L., Hofferbert R., Rehfeld H., Richter A., Schardt P., Decay of classical chaotic systems: The case of the Bunimovich stadium, Phys. Rev. E, 1996, V.53, 2217-2222.

[25] Bunimovich L.A., On the rate of decay of correlations in dynamical systems with chaotic behavior, Sov. Phys. JETP, 1985, V.62, 842-852.

[26] Fendrik A.J., Sánchez M.J., Decay of the Sinai well in D dimensions, Phys. Rev. E, 1995, V.51, $2996-3001$.

[27] Freidman B., Martin R. F., Decay of the velocity autocorrelation function for the periodic Lorentz gas, Phys. Lett. A, 1984, V.105, 23-26.

[28] Artuso R., Casati G., Guarneri I., Numerical experiments on billiards, J. Stat. Phys., 1996, V.83, $145-167$. 